\title{
Low-complexity quantize-and-forward cooperative communication using two-way relaying
}

\author{
lancu Avram ${ }^{*}$, Nico Aerts and Marc Moeneclaey
}

\begin{abstract}
Cooperative communication is used as an effective measure against fading in wireless communication systems. In a classical one-way cooperative system, the relay needs as many orthogonal channels as the number of terminal it assists, yielding a poor spectral efficiency. Efficiency is improved in two-way relaying systems, where a relay simultaneously assists two terminals using only one timeslot. In the current contribution, a two-way quantize-and-forward (QF) protocol is presented. Because of the coarse quantization, the proposed protocol has a low complexity at the relay and can be used with half-duplex devices, making it very suitable for low-complexity applications like sensor networks. Additionally, channel parameter estimation is discussed. By estimating all channel parameters at the destination terminals, relay complexity is kept low. Using Monte Carlo simulations, it is shown that the proposed QF protocol achieves a good frame error rate (FER) performance as compared to two-way amplify-and-forward (AF) and one-way relaying systems. It is further shown that, using the proposed estimation algorithm, the FER degradation arising from the channel parameter estimation is negligible when compared to an (unrealistic) system in which all parameters are assumed to be known.
\end{abstract}

Keywords: Cooperative communication; Two-way relaying; Estimation; Sensor networks; Diversity

\section{Introduction}

Cooperative telecommunication systems can effectively be used to combat fading by exploiting the broadcast nature of the wireless medium [1-6]. In a classical cooperative communication system, only unilateral communication is considered: one transmitting terminal communicates to one receiving terminal with the help of a relaying terminal. Many practical applications however require bilateral communication, in which two terminals both send and receive information to/from each other. Using a classical (one-way) cooperative system in this situation would yield a poor spectral efficiency, as this would require four orthogonal channels, i.e., the two transmitting terminals need one channel each, and the relay transmits over two channels that the data received from the first and second terminal, respectively. The spectral efficiency can be improved using a two-way relaying system,

*Correspondence: iancu.avram@telin.ugent.be

Department of Telecommunications and Information Processing (TELIN, UGent), St-Pietersnieuwstraat 41, 9000 Ghent, Belgium in which the relay uses a single channel to simultaneously assist in the information transfer from the first to the second terminal and from the second to the first terminal.

As for one-way cooperative systems, a variety of forwarding protocols have been developed for two-way systems, including, but not limited to, network coding $[7,8]$, amplify-and-forward (AF) [9], decode-and-forward (DF) $[9,10]$, and compress-and-forward (CF) [11]. While many of these protocols achieve satisfactory results regarding outage probability and frame error rate (FER), they also impose a (large) burden upon the relay in terms of computational complexity and/or storage space requirements. The DF strategy requires the relay to decode the received data. In the AF protocol, the relay needs to store the analog signals awaiting retransmission, requiring a highprecision analog-to-digital conversion (i.e., many quantization bits per sample) and, therefore, a large memory to store the samples.

Two-way quantize-and-forward (QF) protocols have been studied in [12] and [13]. In [13], the capacity of a

\section{是 Springer}

(c) 2014 Avram et al: licensee Springer. This is an Open Access article distributed under the terms of the Creative Commons Attribution License (http://creativecommons.org/licenses/by/4.0), which permits unrestricted use, distribution, and reproduction in any medium, provided the original work is properly credited. 
two-way relaying channel is maximized using an information theoretical approach. Channel symmetry is assumed, i.e. both users' channel qualities need to be the same, both in the uplink and downlink. In [12], a two-way QF relaying scheme using space-time block coding (STBC) is proposed. The two transmitting nodes use STBC to simultaneously transmit their signals to the relay, where they are estimated by using minimum mean square error-ordered successive interference cancellation (MMSE-OSIC). The main drawback of the proposed system is the MMSEOSIC algorithm that needs to be executed at the relay, inevitably raising its complexity. Furthermore, the relay is required to have multiple antenna's, also raising its hardware cost. Both [12] and [13] also assume that there is no direct link between the two user terminals, making it impossible to exploit cooperative diversity.

These hardware requirements can limit the usefulness of existing two-way relaying strategies in applications requiring a low relay complexity, such as sensor networks and battery powered devices. Therefore, in the current contribution, a low-complexity two-way relaying strategy is presented, based on the QF protocol. The main goal is to keep the relay complexity to a minimum by shifting as much operations as possible to the user terminals, where typically there is more processing power available. While QF protocols with a low relay-side complexity have been developed for one-way relaying systems $[14,15]$, the adaptation of these protocols to two-way relaying systems is not straightforward. In the current paper, a novel two-way QF protocol is introduced and its performance is analyzed. The proposed protocol exploits cooperative diversity, by assuming there is a direct path between the user terminals. Furthermore, a practical estimation scheme is proposed for the estimation of all the unknown channel parameters. In order to limit the relay complexity to a minimum, all estimation is performed at the destination terminals, with no additional calculations needed at the relay.

The remainder of this paper is organized as follows: The channel model is outlined in Section 2, whereafter the proposed quantization scheme is presented in Section 3. In Section 4, the receiver structure is obtained and in Section 5, the estimation algorithm is discussed. The FER performance of the proposed algorithms is analyzed using Monte Carlo (MC) simulations in Section 6. Finally, conclusions are drawn in Section 7.

\section{Notations}

Bold lower- and uppercase letters are used to denote vectors and matrices, respectively. The absolute value and phase of the complex number $x$ are denoted as $|x|$ and $\arg (x) \in(0,2 \pi)$, respectively. The Hermitian transpose of $\boldsymbol{x}$ is denoted $\boldsymbol{x}^{H}$ and the suffix $\bmod M$ is used to denote the modulo- $M$ reduction to the interval $[0, M)$. The notation $p(x \mid y ; z)$ refers to the probability density function (pdf) of the random variable $x$, conditioned on the random variable $y$, with $z$ denoting a known deterministic parameter of the considered pdf.

\section{Channel model}

In this contribution, a cooperative two-way relaying scheme is analyzed consisting of two terminals exchanging information, denoted $T_{0}$ and $T_{1}$, and one assisting relay, denoted $R$. At both $T_{0}$ and $T_{1}$, the information to be transmitted is divided into frames of $K$-coded bits, which are obtained by encoding the information bits by means of a channel encoder. The terminals $T_{0}, T_{1}$, and $R$ transmit in turn using time-division multiple access (TDMA), as depicted in Figure 1. At $T_{1}\left(T_{0}\right)$, the signals received from the relay and from $T_{0}\left(T_{1}\right)$ are combined in order to retrieve the information sent by $T_{0}\left(T_{1}\right)$.

All channels are modelled as flat Rayleigh fading channels with additive white Gaussian noise. We denote the complex-valued channel gains between $T_{0}$ and $T_{1}$, between $T_{0}$ and $R$, and between $R$ and $T_{1}$ as $h_{0}, h_{1}$, and $h_{2}$, respectively. The gains of the reciprocal channels are denoted $h_{0}^{\prime}, h_{1}^{\prime}$, and $h_{2}^{\prime}$, respectively, as also shown in Figure 1. In order to keep the discussion general, no assumptions are made on the relation between the fading gains of reciprocal channels. Denoting by $\boldsymbol{c}_{0}, \boldsymbol{c}_{1}$, and $\boldsymbol{c}_{r}$, the

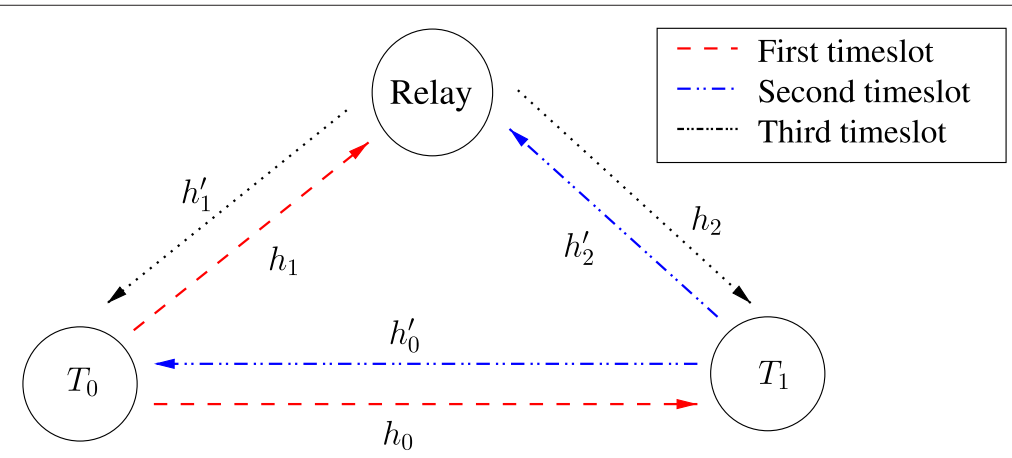

Figure 1 Timeslot assignment. 
PSK symbol sequences sent by $T_{0}$ (first slot), $T_{1}$ (second slot), and $R$ (third slot), respectively, the channel outputs for the information transfer from $T_{0}$ to $T_{1}$ are equal to

$$
\begin{aligned}
& \boldsymbol{r}_{0}=\sqrt{E_{0}} h_{0} \boldsymbol{c}_{0}+\boldsymbol{n}_{0} \\
& \boldsymbol{r}_{1}=\sqrt{E_{0}} h_{1} \boldsymbol{c}_{0}+\boldsymbol{n}_{1} \\
& \boldsymbol{r}_{2}=\sqrt{E_{r}} h_{2} \boldsymbol{c}_{r}+\boldsymbol{n}_{2},
\end{aligned}
$$

where $\boldsymbol{r}_{0}, \boldsymbol{r}_{1}$, and $\boldsymbol{r}_{2}$ denote the signals received by $T_{1}$ (first slot), $R$ (first slot), and $T_{1}$ (third slot), respectively. Similar expressions hold for the reciprocal signals. Assuming the normalization condition $\left|c_{0}\right|^{2}=\left|\boldsymbol{c}_{1}\right|^{2}=\left|\boldsymbol{c}_{r}\right|^{2}=$ $K$, the quantities $E_{0}, E_{1}$, and $E_{r}$ denote the transmitted energy per symbol at $T_{0}, T_{1}$, and $R$, respectively. All channel coefficients are considered to be constant during a frame and have a zero-mean circular symmetric complex Gaussian (ZMCSCG) distribution with variances $N_{h_{x}}=1 / d_{x}{ }^{{ }_{\text {loss }}}, x \in\{0,1,2\}$. The quantity $d_{x}$ represents the distance between the two considered terminals, while $n_{\text {loss }}$ denotes the path loss exponent. The components of the noise vectors $\boldsymbol{n}_{x}$ are also ZMCSCG distributed with variances $N_{x}, x \in\{0,1,2\}$.

\section{Two-way relaying}

In the following subsections, the operation performed at the relay is discussed, assuming that the symbols transmitted by $T_{0}$ and $T_{1}$ belong to a $M_{1}$-PSK constellation, with $M_{1}$ as the constellation size. This operation results in the symbol vector $\boldsymbol{c}_{r}$ transmitted by the relay in the third slot. An AF two-way relaying strategy, to be used for benchmarking the performance of the proposed QF system, is briefly discussed first.

\subsection{Amplify-and-forward}

In a two-way relaying AF system, the relay simply adds the signals received from $T_{0}$ and $T_{1}$ and transmits a scaled version of the resulting sum. This yields the following expression for $\boldsymbol{c}_{r}$ :

$$
\begin{aligned}
\boldsymbol{c}_{r} & =\beta\left(\boldsymbol{r}_{1}+\boldsymbol{r}_{2}^{\prime}\right) \\
& =\beta\left(\sqrt{E_{0}} h_{1} \boldsymbol{c}_{0}+\sqrt{E_{1}} h_{2}^{\prime} \boldsymbol{c}_{1}\right)+\beta\left(\boldsymbol{n}_{1}+\boldsymbol{n}_{2}^{\prime}\right)
\end{aligned}
$$

In order to satisfy the normalization constraint $E\left[\left|\boldsymbol{c}_{r}\right|^{2}\right]=K$, the gain $\beta$ is chosen as

$$
\beta=\frac{1}{\sqrt{E_{0}\left|h_{1}\right|^{2}+E_{1}\left|h_{2}^{\prime}\right|^{2}+N_{1}+N_{2}^{\prime}}} .
$$

Note that the relay in a two-way AF system needs to know the squared channel magnitudes $\left|h_{1}\right|^{2}$ and $\left|h_{2}\right|^{2}$.

\subsection{Quantize-and-forward}

A straightforward implementation of a two-way QF relaying system that is similar to the AF relaying system would involve the coarse quantization of the sum of the signals received in the first and second slot from $T_{0}$ and $T_{1}$, respectively, and the broadcasting of these quantized samples in the third slot. While the initial purpose of quantization is to avoid the storage of analog samples, this approach however would require the relay to store the analog samples received from $T_{0}$ in the first slot, until the data from $T_{1}$ is received in the second slot and the two can be added and quantized. Instead, a quantization scheme that does not necessitate the storage of analog values is proposed, where the relay separately quantizes the signals received in the first and second slot and then properly combines the quantized values. This involves the following operations, which do not require any channel knowledge at the relay.

\subsubsection{Quantization}

In the first and second slot, the phase of the samples received from $T_{0}$ and $T_{1}$, respectively, is quantized uniformly using $\log _{2} M_{2}$ bits. When taking $M_{2} \geq 2 M_{1}$, this approach has shown to yield a performance close to that of AF for one-way relaying systems [14]. The uniform quantization, with $\log _{2} M_{2}$ bits, of the phases of the signals $r_{1}(k)$ and $r_{2}^{\prime}(k)$ received by the relay yields the quantized phases $2 \pi q_{1}(k) / M_{2}$ and $2 \pi q_{2}^{\prime}(k) / M_{2}$, respectively, where

$$
\begin{aligned}
& q_{1}(k)=f_{q}\left(r_{1}(k)\right) \\
& q_{2}^{\prime}(k)=f_{q}\left(r_{2}^{\prime}(k)\right),
\end{aligned}
$$

and the function $f_{Q}(x) \in\left\{0,1, \ldots, M_{2}-1\right\}$ is defined as

$$
f_{Q}(x)=\left\lfloor 0.5+\frac{M_{2}}{2 \pi} \arg (x)\right\rfloor \bmod M_{2} .
$$

so that $f_{Q}(x)=q$ when $\frac{2 \pi}{M_{2}}\left(q-\frac{1}{2}\right) \leq \arg (x)<\frac{2 \pi}{M_{2}}\left(q+\frac{1}{2}\right)$ for $q=1,2, \ldots, M_{2}-1$, and $f_{Q}(x)=0$ when $0 \leq$ $\arg (x)<\frac{\pi}{M_{2}}$ or $2 \pi-\frac{\pi}{M_{2}} \leq \arg (x)<2 \pi$. In order to be able to exploit circular symmetry at the relay, we impose that $M_{2}$ is a multiple of $M_{1}$.

\subsubsection{Addition}

The quantized phases of $r_{1}(k)$ and $r_{2}^{\prime}(k)$ are added, and the resulting sum determines the symbol $c_{r}(k)$ to be sent by the relay in the third slot. Introducing the mapping function

$$
\chi_{M}(q)=\exp \left(\frac{j 2 \pi q}{M}\right),
$$

the symbols sent by the relay can be written as

$$
c_{r}(k)=\chi_{M_{2}}\left(\left(q_{1}(k)+q_{2}^{\prime}(k)\right) \bmod M_{2}\right) .
$$

\subsubsection{Relay complexity}

In the proposed quantization scheme, the storage and processing requirements at the relay are kept low. For each frame, only the vectors $\boldsymbol{q}_{1}$ and $\boldsymbol{q}_{2}^{\prime}$ need to be stored at the relay; the memory requirements are low, because 
the components of $\boldsymbol{q}_{1}$ and $\boldsymbol{q}_{2}^{\prime}$ are represented by only $\log _{2} M_{2}$ bits. The memory usage can further be lowered in a practical implementation by storing the elements of $\boldsymbol{q}_{1}$ obtained in the first slot, performing the modulo $M_{2}$ addition element-wise in the second slot as the values of $\boldsymbol{q}_{2}^{\prime}$ become available by quantizing the incoming signal $\boldsymbol{r}_{2}^{\prime}$, and storing the result of the addition back in $\boldsymbol{q}_{1}$. The latter is then mapped on $M_{2}$-PSK symbols using Equation 2 and broadcast in the third slot.

The number of computations that the relay needs to perform is also limited. The quantization operation has a low complexity, as only the phase of the incoming signals is quantized, neglecting the amplitude. This complexity is further lowered by only considering uniform quantization. The modulo- $M_{2}$ addition of the resulting quantization intervals involves the addition of two integers with a limited range and is thus easily implemented in hardware. Channel parameter estimation does not add to the computational burden of the relay, because all channel parameters are estimated at the destination terminals.

\section{Likelihood calculation}

At $T_{1}\left(T_{0}\right)$, the signals received from the relay and from $T_{0}\left(T_{1}\right)$ need to be combined in order to optimally retrieve the information bits sent by $T_{0}\left(T_{1}\right)$. In this section, we will focus on the calculation of the likelihoods of the received symbols at $T_{1}$, which are used by the channel decoder at $T_{1}$ to detect the information bits transmitted by $T_{0}$. Similar expressions are obtained for the symbol likelihoods at $T_{0}$, used to detect the information transmitted by $T_{1}$. The symbol likelihoods at $T_{1}$ are given by

$$
\begin{aligned}
p\left(\boldsymbol{r}_{0}, \boldsymbol{r}_{2} \mid \boldsymbol{c}_{0}, h_{0}, h_{1}, h_{2}, h_{2}^{\prime} ; \boldsymbol{c}_{1}\right)= & p\left(\boldsymbol{r}_{0} \mid \boldsymbol{c}_{0}, h_{0}\right) \\
& \times p\left(\boldsymbol{r}_{2} \mid \boldsymbol{c}_{0}, h_{1}, h_{2}, h_{2}^{\prime} ; \boldsymbol{c}_{1}\right),
\end{aligned}
$$

As $T_{1}$ knows the symbols $\mathbf{c}_{1}$, Equation 3 denotes the likelihood of $\mathbf{c}_{0}$ based on the observations $\mathbf{r}_{0}$ and $\mathbf{r}_{2}$, and $\mathbf{c}_{1}$ is to be considered as a known parameter. Evaluating Equation 3 on a symbol-by-symbol basis and conditioning on the symbols sent by the relay yields

$$
\begin{aligned}
& p\left(\boldsymbol{r}_{0}, \boldsymbol{r}_{2} \mid \boldsymbol{c}_{0}, h_{0}, h_{1}, h_{2}, h_{2}^{\prime} ; \boldsymbol{c}_{1}\right)= \\
& \prod_{k=1}^{K} p\left(r_{0}(k) \mid c_{0}(k), h_{0}\right) \sum_{c_{r}(k)} p\left(r_{2}(k) \mid c_{r}(k), h_{2}\right) \\
& \quad \times p\left(c_{r}(k) \mid c_{0}(k), h_{1}, h_{2}^{\prime} ; c_{1}(k)\right),
\end{aligned}
$$

with

$$
\begin{aligned}
& p\left(r_{0}(k) \mid c_{0}(k), h_{0}\right)=\frac{1}{\pi N_{0}} \exp \left(-\frac{\left|r_{0}(k)-h_{0} c_{0}(k)\right|^{2}}{N_{0}}\right) \\
& p\left(r_{2}(k) \mid c_{r}(k), h_{2}\right)=\frac{1}{\pi N_{2}} \exp \left(-\frac{\left|r_{2}(k)-h_{2} c_{r}(k)\right|^{2}}{N_{2}}\right) .
\end{aligned}
$$

To determine the factor $p\left(c_{r}(k) \mid c_{0}(k), h_{1}, h_{2}^{\prime} ; c_{1}(k)\right)$ in Equation 4, further on referred to as the transition probabilities, one has to take into account that due to the modulo $M_{2}$ addition, there are multiple combinations of $q_{1}(k)$ and $q_{2}^{\prime}(k)$ that all give rise to the same relay symbol $c_{r}(k)$. This yields the following expression for the transition probabilities:

$$
\begin{aligned}
P\left[c_{r}(k)=\chi_{M_{2}}(q) \mid c_{0}(k), h_{1}, h_{2}^{\prime} ; c_{1}(k)\right]= & \\
\sum_{\tilde{q}=0}^{M_{2}-1} P\left[q_{2}^{\prime}(k)\right. & \left.=\tilde{q} \mid h_{2}^{\prime} ; c_{1}(k)\right] P\left[q_{1}(k)\right. \\
& \left.=(q-\tilde{q}) \bmod M_{2} \mid h_{1}, c_{0}(k)\right] .
\end{aligned}
$$

Let us introduce the function $f_{\Theta}$, describing the pdf of the received phase when a symbol of amplitude 1 and phase 0 is sent over an AWGN channel with a signal-tonoise ratio (SNR) equal to $\gamma$ [14], which is given by

$$
\begin{aligned}
f_{\Theta}(\theta ; \gamma)=\frac{1}{2 \pi} & {\left[e^{-\gamma}+\sqrt{\pi \gamma} \cos (\theta) e^{-\gamma \sin ^{2}(\theta)}\right.} \\
& \times \operatorname{erfc}(-\sqrt{\gamma} \cos (\theta))] .
\end{aligned}
$$

Then, the probabilities $p\left(q_{2}^{\prime}(k) \mid h_{2}^{\prime} ; c_{1}(k)\right)$ and $p\left(q_{1}(k) \mid h_{1}\right.$, $\left.c_{0}(k)\right)$ to be used in Equation 5 can be written as

$$
\begin{aligned}
p\left(q_{2}^{\prime}(k) \mid h_{2}^{\prime} ; c_{1}(k)\right)= & \int_{\frac{2 \pi\left(q_{2}^{\prime}(k)-\frac{1}{2}\right)}{M_{2}}}^{\frac{2 \pi\left(q_{2}^{\prime}(k)+\frac{1}{2}\right)}{M_{2}}} \\
& \times f_{\Theta}\left(\theta-\left(\arg \left(c_{1}(k) h_{2}^{\prime}\right) ; \frac{\left|h_{2}^{\prime}\right|^{2}}{N_{2}^{\prime}}\right) \mathrm{d} \theta\right. \\
p\left(q_{1}(k) \mid h_{1}, c_{0}(k)\right)= & \int_{\frac{2 \pi\left(q_{1}(k)-\frac{1}{2}\right)}{M_{2}}}^{\frac{2 \pi\left(q_{1}(k)+\frac{1}{2}\right)}{M_{2}}} \\
& \times f_{\Theta}\left(\theta-\left(\arg \left(c_{0}(k) h_{1}\right) ; \frac{\left|h_{1}\right|^{2}}{N_{1}}\right) \mathrm{d} \theta,\right.
\end{aligned}
$$

which completes the calculation of the symbol likelihoods.

\section{Estimation}

The likelihoods calculated in the previous section depend on the specific realization of the channel coefficients $h_{0}$, $h_{1}, h_{2}$, and $h_{2}^{\prime}$ (for the likelihoods calculated at $T_{0}$ ) and $h_{0}^{\prime}$, $h_{1}^{\prime}, h_{2}^{\prime}$, and $h_{1}$ (for the likelihoods at $T_{1}$ ). As these parameters change between frames and are not $a$ priori known, they need to be estimated before the data decoding can be performed. In the remainder of this section, we will focus on the channel estimation at $T_{1}$ in order to decode the data sent by $T_{0}$. Similar expressions are obtained for the channel estimation at $T_{0}$. 
The channel coefficients that need to be estimated can be divided into two groups: the ones that are directly observed by $T_{1}$ (these are $h_{0}$ and $h_{2}$ ) and the ones that are not (these are $h_{1}$ and $h_{2}^{\prime}$ ). The main difficulty is estimating the parameters that are not directly observed. In order to keep the complexity at the relay terminal low, we deliberately choose not to perform any relay-side estimation. However, due to the quantization performed at the relay, it is quite difficult to estimate the channel coefficients $h_{1}$ and $h_{2}^{\prime}$ at $T_{1}$. Fortunately, this problem can be circumvented by directly estimating the transition probabilities used in Equation 4, so that we no longer need to know the specific values of $h_{1}$ and $h_{2}^{\prime}$. Indeed, in [16], it was shown that the source-relay transition probabilities can be estimated at the destination in a one-way quantize-andforward system. Accurate results were obtained by first estimating the transition probabilities using pilot symbols transmitted by the source and then iteratively refining these pilot-based estimates by also using the a posteriori probabilities of the unknown data symbols in the estimation process.

In the one-way relaying system described in [16], the transition probabilities only depend on the symbols transmitted by $T_{0}$ and on the channel between $T_{0}$ and $R$. However, in the two-way system at hand, they also depend on the symbols sent by $T_{1}$ and on the channel between $T_{1}$ and $R$, which makes the estimation more complex. In order to be able to apply the results from [16] to the considered two-way system, we first group the transition probabilities from Equation 5 into the three-dimensional array $\boldsymbol{T}$, of which the elements are defined as

$$
\begin{aligned}
T(q, m, n)=P\left[c_{r}=\chi_{M_{2}}(q) \mid c_{0}\right. & =\chi_{M_{1}}(m), h_{1}, h_{2}^{\prime} ; \\
c_{1} & \left.=\chi_{M_{1}}(n)\right] .
\end{aligned}
$$

The symbol index $k$ is omitted from Equation 8 because, due to the slow fading nature of communication channels, the elements of $\boldsymbol{T}$ do not depend on the position within a frame. Note that $\boldsymbol{T}$ contains a total of $M_{1} \times M_{2} \times M_{2}$ elements, all of which need to be estimated. For higher-order mapping constellations, it can be a problem to estimate all elements of $\boldsymbol{T}$ individually, as this would require very long frames and a vast number of pilot symbols. Fortunately, the number of elements from $\boldsymbol{T}$ which actually need to be estimated can be reduced to only $M_{2}$ by exploiting the inherent circular symmetry of $\boldsymbol{T}$. Indeed, defining $\bar{t}(q)=T(q, 0,0)$ with $q=0,1, \ldots, M_{2}-1$, it can be easily shown from Equations 5, 6, and 7 that

$$
T(q, m, n)=\bar{t}\left(\left(q-\frac{M_{2}(m+n)}{M_{1}}\right) \bmod M_{2}\right),
$$

which indicates that, for the given $m$ and $n$, the elements $\left\{T(q, m, n), q=0,1, \ldots, M_{2}-1\right\}$ are obtained as a cyclic shift of the vector $\left.\overline{\boldsymbol{t}}=\overline{(t}(0), \ldots, \bar{t}\left(M_{2}-1\right)\right)$ over $(m+n) \frac{M_{2}}{M_{1}}$ positions.
In order to assist the estimation of $\mathbf{h}=\left(h_{0}, h_{2}, \overline{\boldsymbol{t}}\right)$, both $T_{0}$ and $T_{1}$ transmit pilot symbols which are known to both terminals. These pilot symbols are quantized at the relay using the same quantization method as was used for the data symbols. Hence, the relay operation does not need to distinguish between data symbols and pilot symbols. Using the pilot symbols, an initial estimate of $\mathbf{h}$ is be obtained at $T_{1}$. This pilot-based estimate of $\mathbf{h}$ is then iteratively refined using code-aided estimation that exploits also the presence of the unknown data symbols contained in $\mathbf{r}_{0}$ and $\mathbf{r}_{2}$. The reader is referred to Appendix A and [16] for more details regarding this estimation procedure, which makes use of the expectation-maximization (EM) algorithm.

\section{Performance results}

The frame error rate (FER) performance of the proposed protocol is investigated using Monte Carlo simulations. We consider frames of 1,024 information bits, encoded by means of an $(1,13 / 15)_{8}$ RSCC turbo code [17] that is punctured to a rate of $2 / 3$, yielding a total of 1,536 coded bits which are then mapped on binary phase-shift keying (BPSK) symbols $\left(M_{1}=2\right)$. At the relay, 2-bit quantization of the phase of the received samples is used, yielding transmitted relay symbols belonging to a quadrature phase-shift keying (QPSK) constellation $\left(M_{2}=4\right)$. The path loss exponent equals 4 and the distance between $T_{0}$ and $T_{1}$ are considered unity. All symbol energies are considered to be equal $\left(E_{0}=E_{1}=E_{r}\right)$ and all noise variances are also assumed to be equal $\left(N_{0}=N_{0}^{\prime}=N_{1}=\right.$ $N_{1}^{\prime}=N_{2}=N_{2}^{\prime}$ ). In the remainder of this section, performance metrics related to the information transfer from $T_{0}$ to $T_{1}$ are considered. Results for the communication in the opposite direction are obtained by simply interchanging the positions of $T_{0}$ and $T_{1}$.

\subsection{Channels and transition probabilities known}

In this subsection, the FER performance of the proposed two-way relaying system is analyzed under the assumption that the relevant channels and transition probabilities are known at the receiving terminal. This FER performance is compared to that of a non-cooperative system and a classical one-way relaying system. For a fair comparison, we require the three systems to operate at the same spectral efficiency $R_{b} / R_{s}$, with $R_{b}$ and $R_{s}$ denoting the average information bitrate and the symbol rate, respectively. This is achieved by dimensioning the systems as indicated in Figure 2:

- In the two-way relay system, we use three slots to send 1,024 information bits in each direction $(2,048$ information bits in total), yielding a total transmission time of $2,048 / R_{b}$ and a duration of $2,048 /\left(3 R_{b}\right)$ per slot. As stated in the introduction, 


$$
\begin{aligned}
& \begin{array}{ll}
\text { Non-cooperative: } & T_{0} \rightarrow T_{1} \rightarrow T_{0} \\
\cline { 2 - 3 } &
\end{array} \\
& \text { Two-way: } T_{0} \rightarrow T_{1}, R \quad T_{1} \rightarrow T_{0}, R \quad R \rightarrow T_{0}, T_{1} \\
& \begin{array}{l|l|l|l|l|}
\text { One-way: } & T_{0} \rightarrow T_{1}, R & T_{1} \rightarrow T_{0}, R & R \rightarrow T_{0} & R \rightarrow T_{1} \\
\hline
\end{array}
\end{aligned}
$$

Figure 2 Timeslot assignment for one-way and two-way relaying systems.

the turbo code is punctured to a rate of $2 / 3$, yielding 1,536 coded bits (1,536 BPSK symbols) in the first slot and in the second slot and 1,536 QPSK symbols in the third slot (i.e., $3 \times 1536=4,608$ symbols in total). The resulting spectral efficiency is $R_{b} / R_{s}=4 / 9$ information bits per channel use.

- In the non-cooperative system, there are only two slots as no relay is involved in the communication process. Each slot has a duration of $1,024 / R_{b}$, which is $3 / 2$ times the slot duration of the two-way relay system. The spectral efficiency of $R_{b} / R_{s}=4 / 9$ is obtained by puncturing the turbo code to a rate of $4 / 9$ (instead of 6/9 for the two-way relaying systems), yielding 2,304 coded bits (2,304 BPSK symbols) per slot (i.e., $2 \times 2,304=4,608$ symbols in total).

- In the one-way relay system, the relay uses two slots, to forward the information from $T_{0}$ to $T_{1}$ and from $T_{1}$ to $T_{0}$, requiring a total of four slots, each of duration $512 / R_{b}$. The turbo code is punctured to a rate of $8 / 9$ (instead of $6 / 9$ for the two-way relaying systems), yielding 1,152 coded bits (1,152 BPSK symbols) in the first and the third slot and 1,152 QPSK symbols in the second and in the fourth slot (i.e., $4 \times 1,152=4,608$ symbols in total), again resulting in a spectral efficiency of $R_{b} / R_{s}=4 / 9$.

Also note that, while the above three communication systems yield the same spectral efficiency, the relay in a one-way relaying system needs to transmit more symbols (and thus consume more energy) as compared to the relay in a two-way relaying system. Indeed, in a one-way relaying system, the relay is active during two slots, transmitting a total of 2,034 symbols. In a two-way relaying system, the relay is only active during one slot, transmitting a total of 1,536 symbols. This favors the two-way relaying system in applications where low relay energy consumption is required, such as battery-powered sensor networks and on-body relaying networks.

The FER performance of the considered relaying protocols is shown in Figure 3 as a function of the $E_{b} / N_{0}$ ratio. The quantity $E_{b}$ is used to represent the energy needed to transmit (and relay) 1 information bit from $T_{0}$ to $T_{1}$. The relay position is varied uniformly on a line connecting $T_{0}$ and $T_{1}$ (i.e. in each frame, a random relay position is selected). The figure shows that the proposed two-way QF system achieves a good FER performance that is only

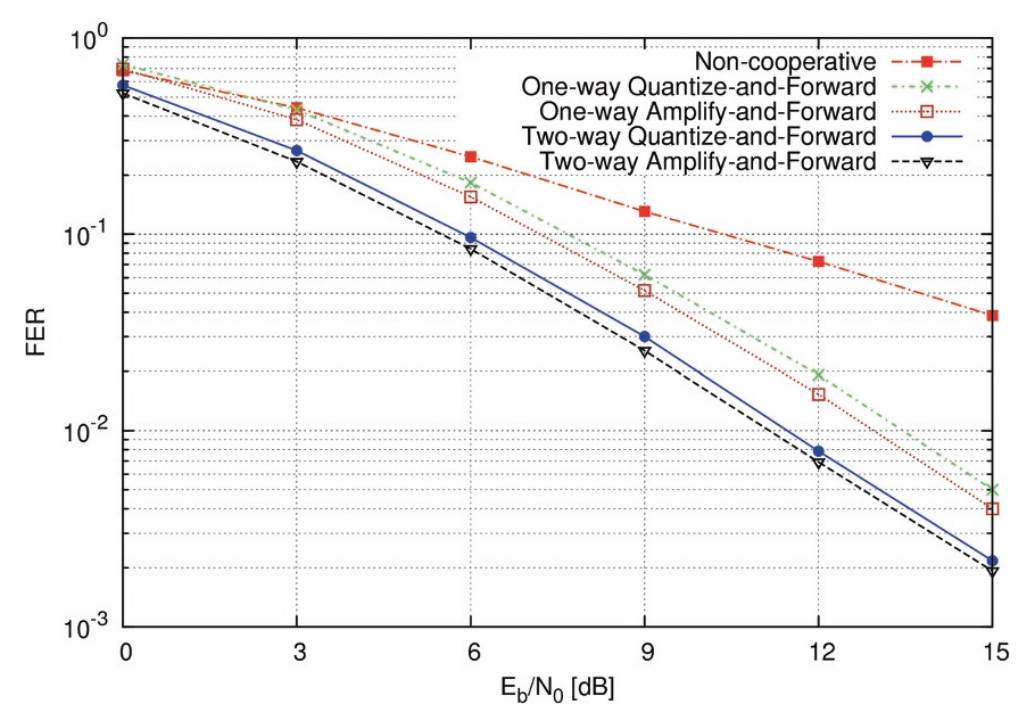

Figure $3 \mathrm{FER}$ as function of the $E_{b} / N_{0}$ ratio. FER of a one-way and a two-way QF and AF protocol along with the FER of a non-cooperative system as function of the $E_{b} / N_{0}$ ratio for a relay position that is uniformly distributed on a line connecting $T_{0}$ and $T_{1}$. 
slightly worse than for the two-way AF system. Both twoway systems outperform their one-way counterparts and due to the increased diversity, all the cooperative systems clearly outperform the non-cooperative system.

In Figure 4, the position of the relay is varied on a line connecting $T_{0}$ and $T_{1}$, while the $E_{b} / N_{0}$ ratio is kept fixed at $9 \mathrm{~dB}$. The resulting FER values are shown as a function of the normalized distance between $T_{0}$ and the relay. In order to better understand the FER behavior of the two-way protocols shown in Figure 4, the FER performance of a two-way AF and QF system in which the relay ignores the signal from $T_{1}$ is also shown. These configurations will be referred to as non-interfering AF and non-interfering QF, respectively. Because the relay ignores the contribution from $T_{1}$, the sole contribution in the signal transmitted by the relay stems from $T_{0}$. In the non-interfering QF (AF) system, the first and third slot support a one-way QF (AF) protocol between $T_{0}$ and $T_{1}$ in which the relay only assists $T_{0}$, while the second slot supports a single-diversity information transfer from $T_{1}$ to $T_{0}$. The following observations can be made from the aforementioned figure:

- In the one-way AF protocol, the FER curve is symmetrical with regard to the relay position. Assuming that $E_{0}\left|h_{1}\right|^{2} \gg N_{1}$ and taking into account the operation of the AF relay, it can easily be verified that signal received from the relay is characterized by an instantaneous SNR given by

$$
\mathrm{SNR}=\left(\frac{N_{1}}{E_{0}\left|h_{1}\right|^{2}}+\frac{N_{2}}{E_{r}\left|h_{2}\right|^{2}}\right)^{-1}
$$

Because $N_{1} / E_{0}=N_{2} / E_{r}$, as specified in the beginning of this section, Equation 10 is symmetrical with respect to $\left|h_{1}\right|^{2}$ and $\left|h_{2}\right|^{2}$, implying the symmetry of the FER curve.

- Due to the coarse quantization at the relay, the one-way QF protocol is outperformed by one-way AF. The degradation of the former with regard to the latter is negligible when the relay is located close to $T_{0}$, but increases when their distance gets larger, because of the decreasing SNR on the $h_{1}$ channel.

- In the two-way AF protocol, the relay transmits a scaled version of the sum of the signals received from $T_{0}$ and $T_{1}$, such that the sum signal has a given energy per symbol interval. As the resulting contribution from $T_{0}$ to the transmitted relay symbols is smaller than in the case of non-interfering AF (in which the contribution from $T_{0}$ is the sole contribution), two-way AF is outperformed by non-interfering AF. The degradation of the two-way AF system with regard to the non-interfering AF system decreases when the relay moves in the direction of $T_{0}$, because in the former system the weight of the signal from $T_{0}$ to the transmitted relay signal increases. When the relay is very close to $T_{0}$, the signal from $T_{1}$ has a negligible contribution to the relay symbols, so the FER performance of the two-way AF system approaches that of the non-interfering AF system.

- In the two-way QF protocol, the symbols transmitted by the relay depend on the phases of the noisy signals received from $T_{0}$ and $T_{1}$ but not on their amplitudes. As the transmitted relay symbols are function of the noise on both the $h_{1}$ and $h_{2}^{\prime}$ channels, they are less

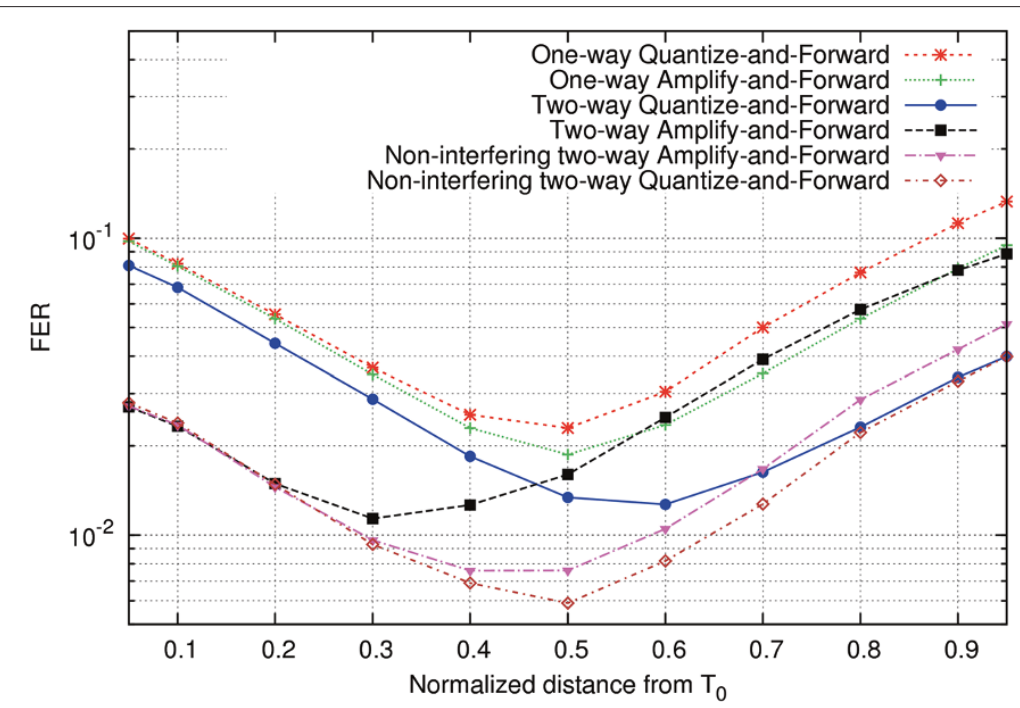

Figure 4 FER as function of the normalized distance between transmitting terminal and relay. FER of a one-way and a two-way QF and AF protocol, along with the FER of a non-interfering two-way QF and AF system as function of the normalized distance between transmitting terminal and relay for a fixed $E_{b} / N_{0}$ ratio of $9 \mathrm{~dB}$. 
reliable than in the non-interfering QF system; therefore, non-interfering QF outperforms two-way QF. The degradation of the two-way QF system with regard to the non-interfering QF system decreases when the relay gets closer to $T_{1}$, because of the increasing SNR on the $h_{2}^{\prime}$ channel. When the distance between the relay and $T_{1}$ is very small, the noise on the $h_{2}^{\prime}$ channel can be ignored, yielding the same situation, and thus the same FER performance, as the non-interfering QF system.

The FER plots from Figure 4 show that, depending on the position of the relay with respect to $T_{0}$ and $T_{1}$, twoway QF clearly outperforms two-way AF and vice versa. When sufficient relay resources are available to support a two-way AF protocol, the results from Figure 4 can be used to determine which protocol is best suited to yield the lowest FER (on average) for the information transfer from $T_{0}$ to $T_{1}$ for a given relay position. Of course, for the same relay position, the selected protocol may not be optimal for the information transfer from $T_{1}$ to $T_{0}$, so trade-offs will have to be made. When we have the freedom to select the position of the relay, we achieve maximum fairness (information transfer from $T_{1}$ to $T_{0}$ and from $T_{0}$ to $T_{1}$ yield the same FER) when the relay is located halfway between $T_{0}$ and $T_{1}$; for this relay position, the two-way QF system slightly outperforms the two-way AF system when $E_{b} / N_{0}=9 \mathrm{~dB}$.

\subsection{Channels and transition probabilities estimated}

Here, we consider the FER performance of the two-way QF relaying system, when the relevant channel gains and transition probabilities are estimated by the destination terminal. In order to assist the estimation, 20 pilot symbols are added to the data frames at both $T_{0}$ and $T_{1}$, so that each slot now consists of 1,556 symbols. The pilot symbols added by $T_{0}$ do not need to equal those added by $T_{1}$, but both pilot symbol sequences need to be known to both terminals. The pilot-based estimates of the transition probabilities and of $h_{2}$ are computed using five EM iterations, while the code-aided refining is executed using eight EM iterations. In the code-aided approach, the EM iterations and turbo decoding iterations are merged as explained in [18]. Using this technique, the increase in complexity induced by the code-aided estimation process is minimal. Figure 5 shows the FER performance of the proposed QF system resulting from pilot-aided estimation and code-aided estimation.

We observe that pilot-based estimation yields a significant degradation with respect to the reference system in which all channel parameters are assumed to be known. This degradation is however almost completely mitigated using the code-aided approach, yielding essentially the same FER performance as the reference system. These results prove that the proposed two-way relaying QF system is suitable to be used in real-life systems, because efficient estimation of the unknown channel parameters can be achieved.

\section{Conclusions}

In this contribution, an implementation has been proposed for a two-way QF relaying system. The computational complexity at the relay has been kept low, in order to make the proposed algorithm suitable for relaying

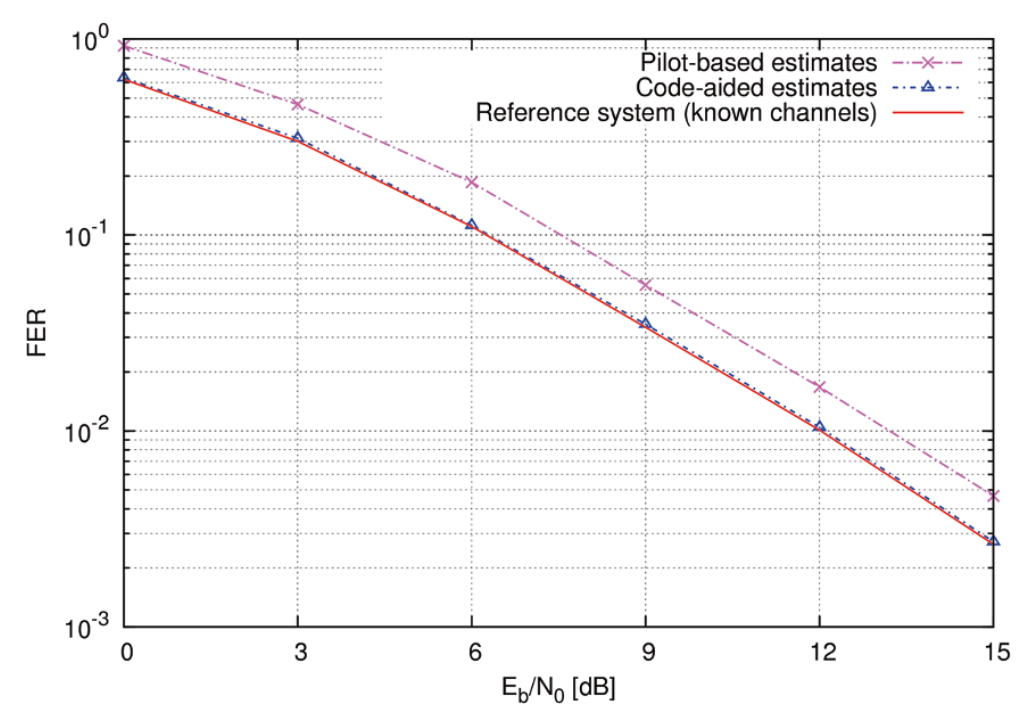

Figure 5 FER performance of a two-way QF system with estimated channel parameters. FER performance of a two-way QF system in which the unknown channel parameters are estimated using different estimation techniques, as function of the $E_{b} / N_{0}$ ratio for a relay position that is uniformly distributed on a line connecting $T_{0}$ and $T_{1}$. 
networks with hardware constraints at the relay, such as sensor networks. After presenting a closed-form expression for the symbol likelihoods at the receivers, the estimation of the unknown channel parameters was discussed. In order to keep the relay complexity low, all parameters are estimated at the destination, requiring no additional operations from the relay. The performance of the proposed algorithms for a relay position that is uniformly distributed between the transmitting and receiving terminal was subsequently evaluated using Monte Carlo simulations. It was shown that the considered two-way QF system clearly outperforms both one-way systems and non-cooperative ones. It was further shown that the FER degradation with respect to a two-way AF system, which has a much higher relay-side complexity, is very low through the full SNR range. In order to gain more insight into the proposed algorithm, the effect of the relay position was also investigated and the results were explained. Finally, it was shown that the proposed estimation algorithms yield only a negligible degradation in FER as compared to an (unrealistic) system in which all channel parameters are assumed to be known, making the presented QF protocol and estimation algorithms suitable to be used in real-life networks that require a low relay-side complexity.

\section{Appendix A}

At $T_{1}$, the channel coefficients $h_{0}$ and $h_{2}$ and the transition probabilities $\overline{\boldsymbol{t}}$ need to be estimated. In [16], channel parameter estimation using the EM algorithm was discussed for a one-way QF system. These results will now be extended to a two-way QF system. The EM algorithm is an iterative algorithm that, besides using the known pilot symbols, also uses the unknown data symbols in the estimation process. These unknown variables are referred to as nuisance parameters. In the case at hand, data symbols transmitted by $T_{0}$ and the symbols transmitted by the relay are considered to be nuisance parameters.

One EM iteration consists of an expectation step and a maximization step. In the expectation step, the estimates from the previous iteration are used to compute the $a$ posteriori expectation of the nuisance parameters. In the maximization step, these expectations are used to update the channel estimates. Introducing $\boldsymbol{h}=\left(h_{0}, h_{2}, \overline{\boldsymbol{t}}\right)$, the expectation step during iteration $i$ consists of calculating the following Q-function

$$
Q\left(\boldsymbol{h}, \hat{\boldsymbol{h}}^{(i-1)}\right)=\mathrm{E}_{\boldsymbol{c}_{s}, \boldsymbol{c}_{r}}\left[\ln p\left(\boldsymbol{r}_{0}, \boldsymbol{r}_{2}, \boldsymbol{c}_{0}, \boldsymbol{c}_{r} \mid \boldsymbol{h} ; \boldsymbol{c}_{1}\right) \mid \boldsymbol{r}_{0}, \boldsymbol{r}_{1}, \hat{\boldsymbol{h}}^{(i-1)}\right]
$$

with $\hat{\boldsymbol{h}}^{(i-1)}$ denoting an estimate of $\boldsymbol{h}$ obtained in the $(i-1)$-th EM iteration. The maximization step involves finding the value of $\boldsymbol{h}$ that maximizes this $\mathrm{Q}$ function, yielding

$$
\hat{\boldsymbol{h}}^{(i)}=\arg \max _{\boldsymbol{h}} Q\left(\boldsymbol{h}, \hat{\boldsymbol{h}}^{(i-1)}\right),
$$

where the value of $\hat{\boldsymbol{h}}^{(0)}$ is initialized using the pilot-based estimates.

Using a similar reasoning as in [16], it can be shown that the elements of $\hat{\boldsymbol{h}}^{(i)}$ are equal to

$$
\begin{aligned}
& {\hat{h_{0}}}^{(i)}=\frac{\boldsymbol{r}_{0} \boldsymbol{u}_{0}^{(i-1)}{ }^{H}}{\left(K+K_{p}\right) E_{0}} \\
& {\hat{h_{2}}}^{(i)}=\frac{\boldsymbol{r}_{2} \boldsymbol{u}_{r}^{(i-1)}{ }^{H}}{\left(K+K_{p}\right) E_{r}} \\
& \hat{t}^{(i)}(q)=\frac{\Gamma^{(i-1)}(q)}{\sum_{\tilde{q}=0}^{M_{2}-1} \Gamma^{(i-1)}(\tilde{q})},
\end{aligned}
$$

with $K_{p}$ the number of pilot symbols and

$$
\begin{gathered}
u_{0}^{(i-1)}(k)=\sum_{m=0}^{M_{1}-1} \chi_{M_{1}}(m) F_{m, n_{0}(k)}^{(i-1)}(k) \\
u_{r}^{(i-1)}(k)=\sum_{q=0}^{M_{2}-1} \sum_{m=0}^{M_{1}-1} \chi_{M_{2}}(q) F_{m, n_{0}(k)}^{(i-1)}(k) \\
. P\left[c_{r}(k)=\chi_{M_{2}}(q) \mid c_{0}(k), \boldsymbol{r}_{2}, \hat{\boldsymbol{h}}^{(i-1)} ; c_{1}(k)\right] \\
\Gamma^{(i-1)}(q)=\sum_{k=1}^{K+K_{p}} \sum_{m=0}^{M_{1}-1} F_{m, n_{0}(k)}^{(i-1)}(k) \\
. P\left[c_{r}(k)=\chi_{M_{2}}\left(q+\frac{M_{2}\left(m+n_{0}\right)}{M_{1}}\right) \mid\right. \\
\left.c_{0}(k)=\chi_{M_{1}}(m), \boldsymbol{r}_{2}, \hat{\boldsymbol{h}}^{(i-1)} ; c_{1}(k)\right]
\end{gathered}
$$

where $n_{0}(k)$ is the integer that satisfies the relation $c_{1}(k)=$ $\chi_{M_{1}}\left(n_{0}(k)\right)$, and $F_{m, n_{0}(k)}^{(i-1)}(k)$ is a short-hand notation for the $a$ posteriori probability of the symbol $c_{0}(k)$ based on the estimate $\hat{\boldsymbol{h}}^{(i-1)}$, i.e.,

$$
\begin{gathered}
F_{m, n}^{(i-1)}(k)=P\left[c_{0}(k)=\chi_{M_{1}}(m) \mid \boldsymbol{r}_{0}, \boldsymbol{r}_{2}, \hat{\boldsymbol{h}}^{(i-1)} ;\right. \\
\left.c_{1}(k)=\chi_{M_{1}}(n)\right]
\end{gathered}
$$

These a posteriori probabilities are provided by the channel decoder at $T_{1}$.

The code-aided EM algorithm is initialized using pilotbased estimates of $\hat{h}_{0}, \hat{h}_{2}$, and $\hat{\boldsymbol{t}}$, denoted as $\hat{h}_{0 p}, \hat{h}_{2 p}$, and $\hat{\boldsymbol{t}}_{p}$, respectively, so that $\hat{h}_{0}^{(0)}=\hat{h}_{0 p}, \hat{h}_{2}^{(0)}=\hat{h}_{2 p}$, and $\hat{\boldsymbol{t}}^{(0)}=\hat{\boldsymbol{t}}_{p}$. These pilot-based estimates are also obtained using the EM algorithm, where only the symbols transmitted by the relay are considered to be nuisance parameters. Denoting the part of the vectors $\boldsymbol{c}_{0}, \boldsymbol{c}_{1}, \boldsymbol{r}_{0}$, and $\boldsymbol{r}_{2}$ that corresponds to 
the pilot symbol positions as $\boldsymbol{c}_{0 p}, \boldsymbol{c}_{1 p}, \boldsymbol{r}_{0 p}$, and $\boldsymbol{r}_{2 p}$, respectively, the pilot-based estimates in the $i$-th EM iteration are given by

$$
\begin{aligned}
& \hat{h}_{0 p}=\frac{\boldsymbol{r}_{0 p} \boldsymbol{c}_{0 p}{ }^{H}}{K_{p} E_{0}} \\
& \hat{h}_{2 p}^{(i)}=\frac{\boldsymbol{r}_{2 p} \boldsymbol{u}_{r p}^{(i-1)}{ }^{H}}{K_{p} E_{r}} \\
& \hat{t}_{p}^{(i)}(q)=\frac{\Gamma_{p}^{(i-1)}(q)}{\sum_{\tilde{q}=0}^{M_{2}-1} \Gamma_{p}^{(i-1)}(\tilde{q})},
\end{aligned}
$$

with

$$
\begin{aligned}
& u_{r p}^{(i-1)}(k)=\sum_{q=0}^{M_{2}-1} \chi_{M_{2}}(q) P\left[c_{r p}(k)=\chi_{M_{2}}(q) \mid \boldsymbol{r}_{2 p},{\hat{h_{2 p}}}^{(i-1)},\right. \\
& \left.\hat{\boldsymbol{\Gamma}}_{p}^{(i-1)} ; c_{0 p}(k), c_{1 p}(k)\right] \\
& \Gamma_{p}^{(i-1)}(q)= \\
& \sum_{k=1}^{K_{p}} P\left[c_{r p}(k)=\chi_{M_{2}}\left(q+\frac{M_{2}\left(m_{0 p}+n_{0 p}\right)}{M_{1}}\right) \mid \boldsymbol{r}_{2 p},{\hat{h_{2 p}}}^{(i-1)},\right. \\
& \left.\hat{\boldsymbol{\Gamma}}_{p}^{(i-1)} ; c_{0 p}(k), c_{1 p}(k)\right]
\end{aligned}
$$

where $m_{0 p}(k)$ and $n_{0 p}(k)$ are the integers that satisfy the relation $c_{0 p}(k)=\chi_{M_{1}}\left(m_{0 p}(k)\right)$ and $c_{1 p}(k)=\chi_{M_{1}}\left(n_{0 p}(k)\right)$, respectively. The initial conditions for the pilot-based EM algorithm are set to $h_{2 p}^{(0)}=1$ and $\Gamma_{p}^{(0)}(q)=1 / M_{2}, \forall q$.

\section{Competing interests}

The authors declare that they have no competing interests.

\section{Acknowledgements}

The authors wish to acknowledge the Agency for Innovation by Science and Technology Flanders (IWT) that motivated this work. This research has been funded by the Interuniversity Attraction Poles Programme initiated by the Belgian Science Policy Office.

Received: 1 August 2014 Accepted: 7 November 2014

Published: 21 November 2014

\section{References}

1. B Sklar, Rayleigh fading channels in mobile digital communication systems part I: characterization. IEEE Commun. Mag. 35(7), 90-100 (1997)

2. TM Cover, JA Thomas, Elements of Information Theory (Wiley, New York, 1991)

3. A Sendonaris, E Erkip, B Aazhang, User cooperation diversity - part I: system description. IEEE Trans. Commun. 51(11), 1927-1938 (2003)

4. TM Cover, A El Gamal, Capacity theorems for the relay channel. IEEE Trans. Inf. Theory 25(5), 572-584 (1979)

5. A El Gamal, M Mohseni, S Zahedi, Bounds on capacity and minimum energy-per-bit for AWGN relay channels. IEEE Trans. Inf. Theory 52(4), 1545-1561 (2006)

6. JN Laneman, Ph. D. thesis, Cooperative diversity in wireless networks: algorithms and architectures. Massachusetts Institute of Technology (2002)

7. $\mathrm{P}$ Larsson, $\mathrm{N}$ Johansson, $\mathrm{K}$ Sunell, Coded bi-directional relaying. IEEE Vehic. Technol. Conf. (VTC 2006-Spring) 2,851-855 (2006)

8. P Popovski, H Yomo, in Proc. IEEE ICC. Physical network coding in two-way wireless relay channels (Glasgow, 2007)
9. B Rankov, A Wittneben, Spectral efficient protocols for half-duplex fading relay channels. IEEE J. Sel. Areas Commun. 25, 379-389 (2007)

10. S Bagheri, F Verde, D Darsena, A Scaglione, Randomized decode-and-forward strategies for two-way relay networks. IEEE Trans. Wireless Commun. 10(12), 4214-4225 (2011)

11. P Zhong, M Vu, in Proc. Allerton Conference on Communication, Control, Computing and Urbana-Champaign, Compress-forward without Wyner-Ziv binning for the one-way and two-Way relay channels, (IL, USA, Sep. 2011), pp. 426-433

12. RM Legnain, RHM Hafez, ID Marand, AM Legnain, in Proc. IEEE Canadian Conference on Electrical \& Computer Engineering (CCECE), Two-way quantize-and-forward relaying with STBC, (Montreal, Canada, 2012), pp. $1-5$

13. M Heindlmaier, O Iscan, C Rosanka, in Proc. IEEE International Symposium on Information Theory (ISIT), Scalar quantize-and-forward for symmetric half-duplex two-way relay channels, (Istanbul, Turkey, 2013), pp. 1322-1326

14. M Souryal, H You, Quantize-and-forward relaying with M-ary phase shift keying. IEEE Wireless Commun. Netw. Conf, 42-47 (2008). doi:10.1109/WCNC.2008.13

15. I Avram, N Aerts, D Duyck, M Moeneclaey, A novel quantize-and-forward cooperative system: channel estimation and m-psk detection performance. EURASIP J. Wireless Commun. Netw. Article ID 415438, 11 (2010)

16. I Avram, N Aerts, H Bruneel, M Moeneclaey, Quantize and forward cooperative communication: channel parameter estimation. IEEE Trans. Wireless Commun. 11(3), 1167-1179 (2012)

17. S Lin, D Costello, Error Control Coding, 2nd edn. (Pearson Education Inc, 2004)

18. N Noels, C Herzet, A Dejonghe, V Lottici, H Steendam, M Moeneclaey, M Luise, L Vandendorpe, in Proc. IEEE ICC, Turbo-synchronization: an EM algorithm approach, (Anchorage, 2003)

doi:10.1186/1687-1499-2014-194

Cite this article as: Avram et al.: Low-complexity quantize-and-forward cooperative communication using two-way relaying. EURASIP Journal on Wireless Communications and Networking 2014 2014:194.

\section{Submit your manuscript to a SpringerOpen ${ }^{\circ}$ journal and benefit from:}

- Convenient online submission

- Rigorous peer review

- Immediate publication on acceptance

- Open access: articles freely available online

- High visibility within the field

- Retaining the copyright to your article

Submit your next manuscript at $>$ springeropen.com 